\title{
Mechanisms of hepatic triglyceride accumulation in non-alcoholic fatty liver disease
}

\author{
Yuki Kawano $\cdot$ David E. Cohen
}

Received: 21 December 2012/ Accepted: 15 January 2013/Published online: 9 February 2013

(C) Springer Japan 2013

\begin{abstract}
Non-alcoholic fatty liver disease (NAFLD) is characterized by hepatic lipid accumulation in the absence of excess alcohol intake. NAFLD is the most common chronic liver disease, and ongoing research efforts are focused on understanding the underlying pathobiology of hepatic steatosis with the anticipation that these efforts will identify novel therapeutic targets. Under physiological conditions, the low steady-state triglyceride concentrations in the liver are attributable to a precise balance between acquisition by uptake of non-esterified fatty acids from the plasma and by de novo lipogenesis, versus triglyceride disposal by fatty acid oxidation and by the secretion of triglyceride-rich lipoproteins. In NAFLD patients, insulin resistance leads to hepatic steatosis by multiple mechanisms. Greater uptake rates of plasma non-esterified fatty acids are attributable to increased release from an expanded mass of adipose tissue as a consequence of diminished insulin responsiveness. Hyperinsulinemia promotes the transcriptional upregulation of genes that promote de novo lipogenesis in the liver. Increased hepatic lipid accumulation is not offset by fatty acid oxidation or by increased secretion rates of triglyceride-rich lipoproteins. This review discusses the molecular mechanisms by which hepatic triglyceride homeostasis is achieved under normal conditions, as well as the metabolic alterations that occur in the setting of insulin resistance and contribute to the pathogenesis of NAFLD.
\end{abstract}

Y. Kawano · D. E. Cohen $(\square)$

Division of Gastroenterology, Department of Medicine,

Brigham and Women's Hospital, Harvard Medical School,

77 Avenue Louis Pasteur, Boston, MA 02115, USA

e-mail: dcohen@partners.org
Keywords Insulin resistance $\cdot$ Fatty acid $\cdot$ Lipid metabolism

\section{Introduction}

Non-alcoholic fatty liver disease (NAFLD) is a major public health issue due to its high prevalence worldwide and potentially serious sequelae. NAFLD is an overarching term for liver histopathology that encompasses a spectrum from simple steatosis to non-alcoholic steatohepatitis (NASH). Whereas simple steatosis is characterized by a relatively favorable clinical course, NASH much more frequently progresses to cirrhosis and hepatocellular carcinoma $[1,2]$. NAFLD is a hepatic manifestation of metabolic syndrome and a risk factor for type 2 diabetes mellitus, dyslipidemia and hypertension [3]. Estimates of the prevalence of NAFLD range widely from 11 to $46 \%$ $[4,5]$. Apart from weight loss, there are currently no effective therapies for NAFLD.

Because the liver does not serve as a storage depot for fat, the steady state concentration of hepatic triglycerides is low under physiological conditions. Nevertheless, there is considerable trafficking of both triglycerides and fatty acids into and out of the liver in response to feeding and fasting. Dietary fatty acids are absorbed from the small intestine, assembled into triglycerides and incorporated into chylomicrons. These are secreted into lymphatics and enter the plasma as triglyceride-rich chylomicrons, where they deliver in excess of $70 \%$ of their fatty acids mainly to adipose tissue, with the remaining being taken up by the liver $[6,7]$. In the setting of excess carbohydrates, fatty acids are also synthesized de novo within the liver [1]. These fatty acids may be converted into other lipid species, such as glycerolipids, glycerophospholipids and sterols, 
which can be packaged into very low density lipoprotein (VLDL) particles and secreted from the liver into the plasma. Under fasting conditions, fatty acids are released from adipose tissue and return to the liver, where they are assembled into triglycerides and packaged to form VLDL particles that are secreted into the plasma. Fatty acids may also be oxidized in situ by mitochondria within the liver.

The hallmark of NAFLD is triglyceride accumulation in the cytoplasm of hepatocytes. This arises from an imbalance between lipid acquisition (i.e., fatty acid uptake and de novo lipogenesis) and removal (i.e., mitochondrial fatty acid oxidation and export as a component of VLDL particles). Here we review the molecular mechanisms that underlie physiological processing of fatty acids and triglycerides by the liver together with the pathophysiological changes that contribute to NAFLD, which are illustrated in Fig. 1.

\section{Fatty acid uptake}

Fatty acid uptake into the liver contributes to the steady balance of hepatic triglycerides in the liver, as well as the pathogenesis of NAFLD. The rate of fatty acid uptake from plasma into cells depends on the fatty acid concentration in plasma and the hepatocellular capacity for fatty acid uptake [8]. Because non-esterified fatty acids are hydrophobic, they are largely bound to albumin in plasma. The process of cellular uptake across the plasma membrane is proteinmediated and requires the dissociation of non-esterified fatty acids from albumin [9]. The cellular capacity for fatty acid uptake depends on the number and activity of transporter proteins on the sinusoidal plasma membrane of the hepatocyte.

The identification and characterization of plasma membrane transporters has remained an area of active

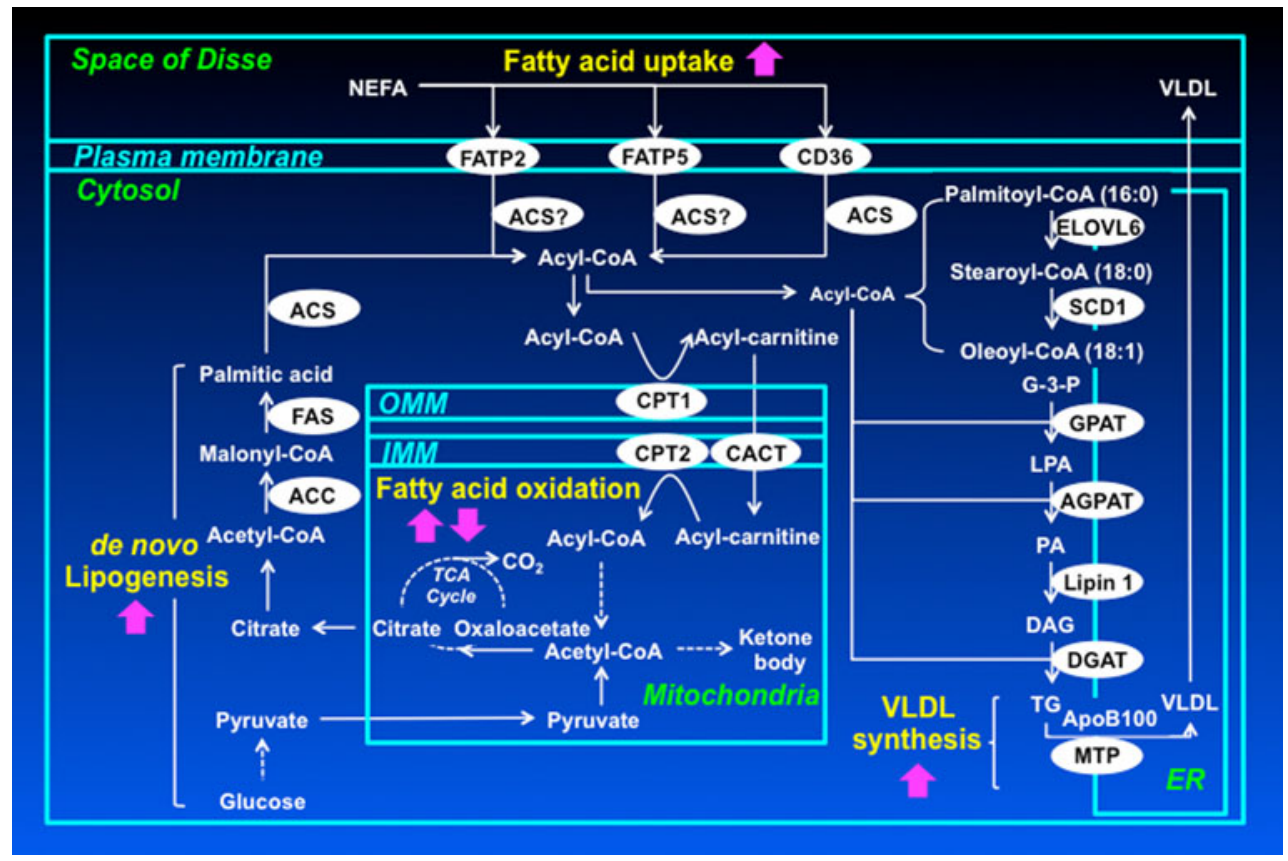

Fig. 1 Mechanisms of hepatocellular lipid metabolism and their dysregulation in non-alcoholic fatty liver disease (NAFLD). Fatty acid uptake: fatty acid transport protein (FATP) 2, FATP5 and CD36 mediate transport of non-esterified fatty acids (NEFA) across the plasma membrane. Once taken up into cytosol, fatty acids are activated to form acyl-CoAs by the activity of FATPs or fatty acylCoA synthetases (ACSs). De novo lipogenesis: palmitic acid is newly synthesized from glucose. Acetyl-CoA carboxylase (ACC) and fatty acid synthase (FAS) catalyze the rate-limiting and final steps, respectively. After ACS-mediated activation, palmitoyl-CoA is elongated by long chain fatty acid elongase 6 (ELOVL6) and desaturated by stearoyl-CoA desaturase 1 (SCD1). Acyl-CoAs are esterified by glycerol-3-phosphate (G-3-P) acyltransferase (GPAT) to form lysophosphatidic acid (LPA) and by 1-acylglycerol-3-phosphate acyltransferase (AGPAT) to form phosphatidic acid (PA). PA is dephosphorylated by lipin 1 to form diacylglycerol (DAG), which is esterified to another acyl-CoA molecule to form triglyceride (TG) by acyl-CoA:diacylglycerol acyltransferase (DGAT). Fatty acid oxidation: acyl-CoAs are transported into mitochondria across the outer mitochondrial membrane (OMM) and inner mitochondrial membrane (IMM) by the activities of carnitine palmitoyl transferase (CPT) 1 , CPT2 and carnitine acylcarnitine translocase (CACT). Within mitochondria, acyl-CoAs are oxidized to form acetyl-CoA. Very low density lipoprotein (VLDL) synthesis: TGs are packaged together with apoB 100 into VLDL in the endoplasmic reticulum (ER) by the activity of microsomal triglyceride transfer protein (MTP) and secreted into space of Disse. Pink arrows denote the increases and decreases that occur in NAFLD and are described in the text. In NAFLD patients, enhanced acquisition of fatty acids through uptake and rates of de novo lipogenesis are not compensated by possible increases in rates of fatty acid oxidation or higher production rates of VLDL particles 
discovery. Fatty acid transport proteins (FATPs) and CD36 (also known as fatty acid translocase, FAT) are key contributors to the transmembrane process. Six FATP isoforms have been identified in mammalian cells $[9,10]$. Of these isoforms, FATP2 is expressed in liver and kidney whereas FATP5 is a liver-specific isoform. In mouse hepatocytes, adenovirus-mediated knockdown of FATP2 or genetic deletion of FATP5 significantly decreases the rates of fatty acid uptake $[11,12]$. CD36 is expressed in a variety of tissues and cell types, muscle and adipose tissue, as well as platelets and macrophages, but its expression level is low in hepatocytes [13]. Mice lacking CD36 exhibit reduced muscle triglyceride contents but also hepatic steatosis [14], supporting the contention that protein-mediated fatty acid uptake to hepatocytes is mainly attributable to FATPs, at least under physiological conditions.

After the entry into cells, fatty acids are rapidly activated by conversion to fatty acyl-CoAs. However, the molecular details of this process remain incompletely understood. One possibility is that FATPs themselves possess fatty acyl-CoA synthetase activity. Purified FATP1 exhibits both long chain and very long chain fatty acyl-CoA synthetase activity [15]. Alternatively, FATPs may interact with long chain acyl-CoA synthetases (ACSLs), which in turn generate the fatty acyl-CoAs. In this connection, FATP1 and ACSL1 were co-immunoprecipitated from 3T3-L1 adipocytes, suggesting that they are interacting proteins [16]. In mammals, five ACSLs have been identified (ACSL1 and $3-6)[17,18]$. In excess of $50 \%$ of the acyl-CoA synthetase activity in the liver is attributable to ACSL1, which is abundant in endoplasmic reticulum (ER) and mitochondria [19]. ACSL5 is also enriched in liver and is mainly localized to mitochondria [20]. Although gain-of-function experiments have demonstrated that ACSL1 and ACSL5 enhance fatty acid uptake in selected cell lines [21, 22], they have no effect on fatty acid uptake rate in hepatocytes, where they appear to channel fatty acids from fatty acid oxidation to lipid synthesis [20]. This is presumably because subcellular distribution of these enzymes differs among cell types. Although it appears unlikely that hepatic ACSLs are coupled with fatty acid uptake, it remains uncertain whether hepatic FATPs are solely responsible for acyl-CoA formation during fatty acid uptake.

Indicative of the importance of fatty acid uptake in NAFLD pathogenesis, Donnelly et al. [7] have demonstrated that approximately $60 \%$ of hepatic triglycerides in human subjects are derived from non-esterified fatty acids in the plasma. This is in line with the observation that plasma non-esterified fatty acid concentrations are elevated in NAFLD subjects, an effect that is attributable to increased fatty acid release from adipose tissue [23, 24]. NAFLD is strongly associated with obesity, and increased fat mass contributes directly to greater fatty acid release from adipose tissue [25]. Peripheral insulin resistance in NAFLD patients also contributes to increased rates of fatty acid release from adipose tissue [26]. Elevated hepatic expression levels of CD36 have been observed in NAFLD and appear to mediate enhanced uptake of non-esterified fatty acids $[27,28]$. A potential pathogenic role is supported by the observation that hepatic overexpression of CD36 in mice increases liver triglyceride contents [29]. Collectively, these findings support a pathogenic role for fatty acid uptake in NAFLD.

Recent studies have also suggested that hepatic uptake of fatty acids via FATPs can be a novel therapeutic strategy for NAFLD. Adenovirus-mediated knockdown of FATP2 or FATP5 reduced hepatic triglyceride accumulation in mice fed a high fat diet $[12,30]$. Moreover, both deoxycholic and ursodeoxycholic acid have shown promise as inhibitors of FATP5-mediated fatty acid uptake, suggesting that bile acid treatment may improve hepatic steatosis, at least in high fat fed mice [31].

\section{Triglyceride synthesis}

The non-esterified fatty acids that are incorporated into triglycerides within liver may be derived from the plasma or be newly synthesized from glucose (so-called "de novo lipogenesis"). De novo lipogenesis plays a substantial role in the pathogenesis of NAFLD, accounting for $26 \%$ hepatic triglycerides in human subjects [7]. Here we describe the mechanisms of hepatic triglyceride accumulation through de novo lipogenesis under physiological conditions followed by their aberrant regulation in NAFLD.

For de novo lipogenesis, glucose is converted to acetylCoA through glycolysis and the oxidation of pyruvate. Acetyl-CoA is then converted to malonyl-CoA by acetylCoA carboxylase (ACC). Fatty acid synthase (FAS) catalyzes the formation of palmitic acid from malonyl-CoA and acetyl-CoA. Palmitic acid is then elongated and desaturated by long chain fatty acid elongase 6 (ELOVL6) and stearoylCoA desaturase 1 (SCD1) to generate monounsaturated fatty acids, which are the major fatty acid constituents of triglycerides. Glycerol-3-phosphate acyltransferase (GPAT) then catalyzes the esterification of glycerol-3-phosphate from glycolysis with the newly synthesized fatty acid to generate lysophosphatidic acids. Lysophosphatidic acids are substrates for 1-acylglycerol-3-phosphate acyltransferase (AGPAT) to catalyze the formation of phosphatidic acids. Phosphatidic acids are then processed to diacylglycerols by lipin 1 [32], followed by the formation of triglycerides through acyl-CoA:diacylglycerol acyltransferase (DGAT).

Lipogenesis is controlled primarily at the transcriptional level $[32,33]$. Postprandially, plasma glucose and insulin concentrations rise, and both are required for full activation 
of lipogenesis [33]. Glucose and insulin promote lipogenesis by activation of carbohydrate response element binding protein (ChREBP) and sterol regulatory element binding protein 1c (SREBP1c), respectively. These are master transcription factors, which in turn promote transcription of lipogenic genes.

SREBP1c is a transcription factor that promotes the expression of lipogenic genes, including FAS, ACC, SCD1 and lipin $1[32,34]$. Insulin promotes the transcriptional upregulation of SREBP1c by an incompletely understood phosphoinositide 3-kinase (PI3K)-dependent mechanism that relies upon the participation of liver X receptor (LXR) [33]. LXR, a nuclear hormone receptor that also plays a key role in regulating cellular cholesterol homeostasis, is activated by oxysterol metabolites of cholesterol [35]. Once activated, LXR heterodimerizes with the retinoid X receptor (RXR) and binds to the LXR response element, leading to upregulation of target genes involved in cholesterol catabolism and elimination [35]. Of the two isoforms of LXR, LXR $\alpha$ is the predominant isoform in liver [35] and a potent activator of SREBP1c [33, 36].

ChREBP also plays an important role in glucose-mediated lipogenesis within the liver. ChREBP is a glucoseresponsive transcription factor that relocates to the nucleus in response to increased glucose concentrations. Glucose promotes dephosphorylation of amino acid residues on ChREBP, and this results in transport into the nucleus and binding to the gene promoters [33]. ChREBP stimulates the expression of lipogenic genes, but also the expression of liver-type pyruvate kinase, thus providing more substrate for fatty acid and triglyceride synthesis [1,37].

The term "selective insulin resistance" has been used to describe the metabolic events within the liver that appear to contribute to NAFLD pathogenesis. Muscle and liver insulin resistance develops in the setting of overnutrition by mechanisms that are complex, but converge to promote the accumulation of specific lipid metabolites (i.e., diacylglycerols and/or ceramides) in liver and skeletal muscle that impair insulin signaling and promote insulin resistance [38]. Notwithstanding its failure to suppress hepatic gluconeogenesis, insulin continues to promote lipogenesis within the liver. Mechanisms of this selective insulin resistance are the subject of active investigation. Within the insulin signaling pathway, the mammalian target of rapamycin complex 1 (mTORC1) and insulin induced gene 2A (INSIG2A) are required for stimulation of lipogenesis [39, 40], but apparently not for inhibition of gluconeogenesis [39], which is regulated by the transcription factor forkhead box O1 (FoxO1). This divergence in responses could explain how glucose and lipid metabolism might be separately controlled within the same pathway. It has also been proposed that ER stress in the context of overnutrition leads to cellular processing and activation of SREBP1c, which promotes lipogenesis and contributes to the development of hepatic steatosis [33].

\section{Fatty acid oxidation}

The steady state balance of hepatic triglycerides is also controlled by the consumption of fatty acids by mitochondrial $\beta$-oxidation [41], which is critical for production of both ATP and ketone bodies. Fatty acyl-CoAs, the activated form of fatty acids, traverse mitochondrial membranes in a carnitine-dependent manner. The presence of an acyl-CoA synthetase in the outer mitochondrial membrane suggests that activation of fatty acids for mitochondrial uptake occurs as an initial step in the transport process. Translocation of fatty acyl-CoAs across the outer mitochondrial membrane is coupled to their conversion to acyl-carnitines by the activity of carnitine palmitoyltransferase (CPT) 1, which is also localized in the outer mitochondrial membrane and catalyzes the formation of acylcarnitine from acyl-CoA and free carnitine. Acyl-carnitines are transported across the inner mitochondrial membrane by carnitine acylcarnitine translocase in exchange for free carnitine. CPT2, which is expressed on the mitochondrial inner membrane, converts acyl-carnitines back to acylCoAs. Within the mitochondrial matrix, acyl-CoAs are sequentially broken down by the $\beta$-oxidation cycle into acetyl-CoAs. This process requires four enzymes: acylCoA dehydrogenase, 2-enoyl-CoA hydratase, 3-hydroxyacyl-CoA dehydrogenase and 3-oxoacyl-CoA thiolase. Acetyl-CoAs are further oxidized in the tricarboxylic acid (TCA) cycle where NADH and $\mathrm{FADH}_{2}$ are generated for ATP synthesis by the electron transport chain. When present in excess, acetyl-CoAs are processed to ketone bodies by a series of reactions for which 3-hydroxy-3methylglutaryl-CoA (HMG-CoA) synthase is the ratelimiting enzyme [42].

In the postprandial state, $\beta$-oxidation in the liver is suppressed. This occurs in part due to the antilipolytic effect of insulin on white adipose tissue, which reduces the flux of non-esterified fatty acid to the liver, and in part due to the direct control by glucose and insulin over the rate of fatty acid entry into the mitochondria [43]. As described above, insulin facilitates de novo lipogenesis through upregulation and activation of SREBP1c and induction of ACC. Malonyl-CoA produced by ACC activity inhibits the activity of CPT1, and thereby decreases the rate of $\beta$-oxidation by reducing fatty acid entry to mitochondria. Cytosolic ACC1, the major isoform of ACC in liver, contributes malonyl-CoA for fatty acid synthesis [44] and for inhibition of fatty acid oxidation [45]. By contrast, malonyl-CoA produced by ACC2, which is localized in mitochondria, serves primarily to suppress fatty acid 
oxidation [46]. These observations are in keeping with regulation of malonyl-CoA as the molecular switch between fatty acid synthesis and oxidation [47].

During the fasting state, glucagon promotes fatty acid oxidation. Glucagon signaling activates AMP-activated protein kinase (AMPK), which in turn inactivates ACC1 and ACC2 by phosphorylation [47]. This blocks the synthesis of malonyl-CoA and is accompanied by activation malonyl-CoA decarboxylase, which enhances destruction of malonyl-CoA [47]. These combined effects decrease fatty acid synthesis and activate ketogenesis. The hepatic expression of peroxisome proliferator-activated receptor (PPAR) $\alpha$ is also essential for glucagon-mediated fatty acid oxidation [48]. PPAR $\alpha$ is a fatty acid-activated nuclear hormone receptor that plays a central role in the transcriptional regulation of lipid and glucose metabolism [49]. Activated PPAR $\alpha$ forms a heterodimer with RXR, which binds to specific DNA sequences known as peroxisome proliferator response elements (PPREs). This transcriptional complex promotes the expression of genes that mediate fatty acid oxidation. Although a common feature of PPAR $\alpha$ target genes, PPREs have not been identified in all genes that are regulated by this transcription factor [49]. Recent studies have demonstrated that the circulating hormone fibroblast growth factor 21 (FGF21) is directly induced by PPAR $\alpha$ [50]. In mice, FGF21 promotes fatty acid oxidation and ketogenesis, as well as gluconeogenesis during fasting. FGF21 is primarily expressed in liver and released to the circulation to function as an endocrine hormone. In humans, the regulatory role of FGF21 in fatty acid oxidation is less clear [51].

Accumulating evidence also indicates that members of the sirtuin gene family, especially SIRT1 and SIRT3, play important roles in fatty acid oxidation during conditions of caloric deprivation [52]. Sirtuins control target proteins by deacetylating histones and other transcriptional regulators. In rodents, both SIRT1 and SIRT3 are increased in response to fasting and activate proteins that promote fatty acid oxidation. SIRT1 enhances fatty acid oxidation by activating PPAR $\alpha$ and its co-activator, peroxisome proliferator-activated receptor-gamma coactivator $1 \alpha$ (PGC1 $\alpha)$. SIRT1 also reduces lipogenesis by inhibiting SREBP1c. SIRT3, which is localized to mitochondria, promotes ß-oxidation by activating acyl-CoA dehydrogenase.

Studies in human NAFLD patients have yielded mixed results with respect to alterations in rates of fatty acid oxidation [38]. Impaired ATP production has been described in patients with NAFLD and insulin resistance [53, 54]. Others have reported evidence for increased rates of fatty acid oxidation in NAFLD $[55,56]$. The latter findings suggest that increased mitochondrial activity could promote oxidative stress within the liver and contribute to the development of NASH [57].

\section{VLDL secretion}

The liver secretes triglycerides in the form of VLDL particles for delivery to peripheral tissues, including skeletal muscle, cardiac muscle and adipose tissue. VLDL is a triglyceride-rich lipoprotein, which contains a hydrophobic core composed mainly of triglycerides and cholesteryl esters. This core is surrounded by a hydrophilic coating, which consists of a monolayer of phospholipids, as well as unesterified cholesterol. These constituents are delivered to peripheral tissues as VLDL is converted by lipoprotein lipase to higher-density and smaller-sized atherogenic particles, including low density lipoproteins (LDL) and intermediate density lipoproteins (IDL). Each VLDL particle is stabilized by a single molecule of apolipoprotein B 100 (apoB 100). ApoB 100 is a long polypeptide that is lipidated with triglycerides within the lumen of ER while it is being translated and translocated across the ER membrane. Lipidation of apoB 100 is facilitated by microsomal triglyceride transfer protein (MTP), an ER resident protein that has both apoB 100 binding and lipid transfer domains [58]. Nascent VLDL particles, packaged into VLDL transport vesicles, are transported from ER to Golgi apparatus [59]. During these processes, the maturing VLDL particles are further lipidated by the activity of MTP [60]. Upon maturation, VLDL particles are exocytosed into the plasma within the space of Disse.

The VLDL secretion rate appears to depend not only on the availability of hepatic triglycerides, but also on the overall capacity for VLDL assembly. When the triglyceride availability is reduced, lipid-free apoB 100 is degraded by both proteasomal and non-proteasomal pathways [61]. Insulin plays an important role in regulating the capacity for VLDL assembly. In response to insulin action, apoB 100 is degraded by mechanisms that appear to include autophagy [61]. Moreover, insulin negatively regulates the expression of MTP, which is a target gene of FoxO1, the same transcription factor responsible for induction of gluconeogenic genes [62]. Nuclear FoxO1 binds to MTP promoter regions and increases transcription [62]. Phosphorylation of FoxO1 in response to insulin signaling prevents its translocation from the cytosol to the nucleus. Although insulin-mediated lipogenesis in the fed state would appear to make more triglycerides be available for VLDL assembly, the net effect of insulin is to limit the postprandial secretion of VLDL. This allows the preferentially delivery of dietary triglycerides to peripheral tissues in the form of apoB 48-containing chylomicron particles.

Impaired VLDL assembly and secretion result in excessive lipid accumulation in liver. Hepatic steatosis has been reported in subjects with hypobetalipoproteinemia (i.e., mutations in apoB 100) [63] or abetalipoproteinemia 
(i.e., mutations in MTP) [64]. Similar observations have been observed in liver-specific MTP knockout mice [65], whereas mice with liver-specific overexpression of MTP exhibit VLDL overproduction and elevated plasma triglyceride levels [66]. Hepatic steatosis may also be induced by drugs that inhibit MTP activity [67]. By contrast, NAFLD is characterized by overproduction of VLDL particles, which reflects enhanced de novo lipogenesis plus lipolysis of intrahepatic and intra-abdominal fat [24]. In these subjects, lipid availability for VLDL assembly is increased, and this is combined with the failure of insulin to suppress VLDL production. Due to the increased availability of triglycerides, apoB 100 is not degraded, and MTP expression is increased due to sustained nuclear localization of FoxO1 [68]. Consequently, hypertriglyceridemia is a common finding in NAFLD patients. Although chronic exposure to insulin drives VLDL overproduction [68], augmented VLDL secretion does not compensate the triglyceride overproduction in liver, so that steady state hepatic triglyceride concentrations remain increased. Moreover, apoB 100 secretion is not increased in NAFLD, suggesting that apoB 100 production limits the liver's capacity to export hepatic triglycerides [24]. In this connection, prolonged exposure of the liver to non-esterified fatty acids, such as that occuring in the setting of insulin resistance, promotes excessive ER stress, and this leads to the degradation of apoB 100, thereby decreasing triglyceride secretion and worsening steatosis [69].

\section{Conclusions}

Under physiological conditions, the delicate balance between accumulation and disposal results in low steady state triglyceride concentrations within the liver. In the setting of overnutrition and insulin resistance, this balance is altered by a multiplicity of events that increases hepatic triglyceride concentrations and results in steatosis. Although rare genetic defects in fatty acid oxidation- or VLDL secretion-related genes may induce hepatic steatosis in humans, more common human genetic variants have now been linked to NAFLD susceptibility [1]. However, the roles of these genes in the pathogenesis of hepatic steatosis are not well understood. The I148M missense mutation in patatin-like phospholipase domaincontaining (PNPLA) 3 is most consistently associated with NAFLD. Indicative of its complex regulatory role in hepatic triglyceride metabolism, overexpression of PNPLA3 in a transgenic mouse model promoted NAFLD in the absence of insulin resistance by increasing the formation of fatty acids and triglycerides, while impairing triglyceride hydrolysis [70]. Additional studies on PNPLA3 and other genes linked to NAFLD should advance our understanding of the molecular pathogenesis of this common disorder.

Acknowledgments This study was supported by National Institutes of Health grants DK56626 and DK48873 to D.E.C.

Conflict of interest Dr. Yuki Kawano declares that he has no conflict of interest. Dr. David Cohen serves as a consultant to Genzyme and Merck.

\section{References}

1. Cohen JC, Horton JD, Hobbs HH. Human fatty liver disease: old questions and new insights. Science. 2011;332:1519-23.

2. Starley BQ, Calcagno CJ, Harrison SA. Nonalcoholic fatty liver disease and hepatocellular carcinoma: a weighty connection. Hepatology. 2010;51:1820-32.

3. Adams LA, Waters OR, Knuiman MW, Elliott RR, Olynyk JK. NAFLD as a risk factor for the development of diabetes and the metabolic syndrome: an eleven-year follow-up study. Am J Gastroenterol. 2009;104:861-7.

4. Lazo M, Clark JM. The epidemiology of nonalcoholic fatty liver disease: a global perspective. Semin Liver Dis. 2008;28:339-50.

5. Williams CD, Stengel J, Asike MI, Torres DM, Shaw J, Contreras $\mathrm{M}$, et al. Prevalence of nonalcoholic fatty liver disease and nonalcoholic steatohepatitis among a largely middle-aged population utilizing ultrasound and liver biopsy: a prospective study. Gastroenterology. 2011;140:124-31.

6. Nestel PJ, Havel RJ, Bezman A. Sites of initial removal of chylomicron triglyceride fatty acids from the blood. J Clin Invest. 1962;41:1915-21.

7. Donnelly KL, Smith CI, Schwarzenberg SJ, Jessurun J, Boldt MD, Parks EJ. Sources of fatty acids stored in liver and secreted via lipoproteins in patients with nonalcoholic fatty liver disease. J Clin Invest. 2005;115:1343-51.

8. Bradbury MW. Lipid metabolism and liver inflammation. I. Hepatic fatty acid uptake: possible role in steatosis. Am J Physiol Gastrointest Liver Physiol. 2006;290:G194-8.

9. Doege H, Stahl A. Protein-mediated fatty acid uptake: novel insights from in vivo models. Physiology (Bethesda). 2006;21: 259-68.

10. Hirsch D, Stahl A, Lodish HF. A family of fatty acid transporters conserved from mycobacterium to man. Proc Natl Acad Sci USA. 1998;95:8625-9.

11. Doege H, Baillie RA, Ortegon AM, Tsang B, Wu Q, Punreddy S, et al. Targeted deletion of FATP5 reveals multiple functions in liver metabolism: alterations in hepatic lipid homeostasis. Gastroenterology. 2006;130:1245-58.

12. Falcon A, Doege H, Fluitt A, Tsang B, Watson N, Kay MA, et al. FATP2 is a hepatic fatty acid transporter and peroxisomal very long-chain acyl-CoA synthetase. Am J Physiol Endocrinol Metab. 2010;299:E384-93.

13. Su X, Abumrad NA. Cellular fatty acid uptake: a pathway under construction. Trends Endocrinol Metab. 2009;20:72-7.

14. Hajri T, Han XX, Bonen A, Abumrad NA. Defective fatty acid uptake modulates insulin responsiveness and metabolic responses to diet in CD36-null mice. J Clin Invest. 2002;109:1381-9.

15. Hall AM, Smith AJ, Bernlohr DA. Characterization of the AcylCoA synthetase activity of purified murine fatty acid transport protein 1. J Biol Chem. 2003;278:43008-13.

16. Richards MR, Harp JD, Ory DS, Schaffer JE. Fatty acid transport protein 1 and long-chain acyl coenzyme A synthetase 1 interact in adipocytes. J Lipid Res. 2006;47:665-72. 
17. Li LO, Klett EL, Coleman RA. Acyl-CoA synthesis, lipid metabolism and lipotoxicity. Biochim Biophys Acta. 2010;1801: 246-51.

18. Soupene E, Kuypers FA. Mammalian long-chain acyl-CoA synthetases. Exp Biol Med (Maywood). 2008;233:507-21.

19. Li LO, Ellis JM, Paich HA, Wang S, Gong N, Altshuller G, et al. Liver-specific loss of long chain acyl-CoA synthetase-1 decreases triacylglycerol synthesis and beta-oxidation and alters phospholipid fatty acid composition. J Biol Chem. 2009;284:27816-26.

20. Bu SY, Mashek DG. Hepatic long-chain acyl-CoA synthetase 5 mediates fatty acid channeling between anabolic and catabolic pathways. J Lipid Res. 2010;51:3270-80.

21. Mashek DG, McKenzie MA, Van Horn CG, Coleman RA. Rat long chain acyl-CoA synthetase 5 increases fatty acid uptake and partitioning to cellular triacylglycerol in McArdle-RH7777 cells. J Biol Chem. 2006;281:945-50.

22. Milger K, Herrmann T, Becker C, Gotthardt D, Zickwolf J, Ehehalt R, et al. Cellular uptake of fatty acids driven by the ERlocalized acyl-CoA synthetase FATP4. J Cell Sci. 2006;119: 4678-88.

23. Holt HB, Wild SH, Wood PJ, Zhang J, Darekar AA, Dewbury K, et al. Non-esterified fatty acid concentrations are independently associated with hepatic steatosis in obese subjects. Diabetologia. 2006; 49:141-8.

24. Fabbrini E, Mohammed BS, Magkos F, Korenblat KM, Patterson BW, Klein S. Alterations in adipose tissue and hepatic lipid kinetics in obese men and women with nonalcoholic fatty liver disease. Gastroenterology. 2008;134:424-31.

25. Fabbrini E, Sullivan S, Klein S. Obesity and nonalcoholic fatty liver disease: biochemical, metabolic, and clinical implications. Hepatology. 2010;51:679-89.

26. Hwang JH, Stein DT, Barzilai N, Cui MH, Tonelli J, Kishore P, et al. Increased intrahepatic triglyceride is associated with peripheral insulin resistance: in vivo MR imaging and spectroscopy studies. Am J Physiol Endocrinol Metab. 2007;293:E1663-9.

27. Greco D, Kotronen A, Westerbacka J, Puig O, Arkkila P, Kiviluoto $\mathrm{T}$, et al. Gene expression in human NAFLD. Am J Physiol Gastrointest Liver Physiol. 2008;294:G1281-7.

28. Miquilena-Colina ME, Lima-Cabello E, Sanchez-Campos S, Garcia-Mediavilla MV, Fernandez-Bermejo M, Lozano-Rodriguez T, et al. Hepatic fatty acid translocase CD36 upregulation is associated with insulin resistance, hyperinsulinaemia and increased steatosis in non-alcoholic steatohepatitis and chronic hepatitis C. Gut. 2011;60:1394-402.

29. Koonen DP, Jacobs RL, Febbraio M, Young ME, Soltys CL, Ong $\mathrm{H}$, et al. Increased hepatic CD36 expression contributes to dyslipidemia associated with diet-induced obesity. Diabetes. 2007;56:2863-71.

30. Doege H, Grimm D, Falcon A, Tsang B, Storm TA, Xu H, et al. Silencing of hepatic fatty acid transporter protein 5 in vivo reverses diet-induced non-alcoholic fatty liver disease and improves hyperglycemia. J Biol Chem. 2008;283:22186-92.

31. Nie B, Park HM, Kazantzis M, Lin M, Henkin A, Ng S, et al. Specific bile acids inhibit hepatic fatty acid uptake in mice. Hepatology. 2012;56:1300-10.

32. Csaki LS, Reue K. Lipins: multifunctional lipid metabolism proteins. Annu Rev Nutr. 2010;30:257-72.

33. Ferre P, Foufelle F. Hepatic steatosis: a role for de novo lipogenesis and the transcription factor SREBP-1c. Diabetes Obes Metab. 2010;12(Suppl 2):83-92.

34. Horton JD, Goldstein JL, Brown MS. SREBPs: activators of the complete program of cholesterol and fatty acid synthesis in the liver. J Clin Invest. 2002;109:1125-31.

35. Chawla A, Repa JJ, Evans RM, Mangelsdorf DJ. Nuclear receptors and lipid physiology: opening the X-files. Science. 2001;294:1866-70.
36. Schultz JR, Tu H, Luk A, Repa JJ, Medina JC, Li L, et al. Role of LXRs in control of lipogenesis. Genes Dev. 2000;14:2831-8.

37. Uyeda K, Repa JJ. Carbohydrate response element binding protein, ChREBP, a transcription factor coupling hepatic glucose utilization and lipid synthesis. Cell Metab. 2006;4:107-10.

38. Samuel VT, Shulman GI. Mechanisms for insulin resistance: common threads and missing links. Cell. 2012;148:852-71.

39. Li S, Brown MS, Goldstein JL. Bifurcation of insulin signaling pathway in rat liver: mTORC1 required for stimulation of lipogenesis, but not inhibition of gluconeogenesis. Proc Natl Acad Sci USA. 2010;107:3441-6.

40. Yecies JL, Zhang HH, Menon S, Liu S, Yecies D, Lipovsky AI, et al. Akt stimulates hepatic SREBP1c and lipogenesis through parallel mTORC1-dependent and independent pathways. Cell Metab. 2011;14:21-32.

41. Eaton S, Bartlett K, Pourfarzam M. Mammalian mitochondrial beta-oxidation. Biochem J. 1996;320:345-57.

42. McGarry JD, Foster DW. Regulation of hepatic fatty acid oxidation and ketone body production. Annu Rev Biochem. 1980; 49:395-420.

43. Sidossis LS, Stuart CA, Shulman GI, Lopaschuk GD, Wolfe RR. Glucose plus insulin regulate fat oxidation by controlling the rate of fatty acid entry into the mitochondria. J Clin Invest. 1996;98: 2244-50.

44. Mao J, DeMayo FJ, Li H, Abu-Elheiga L, Gu Z, Shaikenov TE, et al. Liver-specific deletion of acetyl-CoA carboxylase 1 reduces hepatic triglyceride accumulation without affecting glucose homeostasis. Proc Natl Acad Sci USA. 2006;103:8552-7.

45. Savage DB, Choi CS, Samuel VT, Liu ZX, Zhang D, Wang A, et al. Reversal of diet-induced hepatic steatosis and hepatic insulin resistance by antisense oligonucleotide inhibitors of acetyl-CoA carboxylases 1 and 2. J Clin Invest. 2006;116: 817-24.

46. Abu-Elheiga L, Matzuk MM, Abo-Hashema KA, Wakil SJ. Continuous fatty acid oxidation and reduced fat storage in mice lacking acetyl-CoA carboxylase 2. Science. 2001;291:2613-6.

47. Foster DW. Malonyl-CoA: the regulator of fatty acid synthesis and oxidation. J Clin Invest. 2012;122:1958-9.

48. Longuet C, Sinclair EM, Maida A, Baggio LL, Maziarz M, Charron MJ, et al. The glucagon receptor is required for the adaptive metabolic response to fasting. Cell Metab. 2008;8: 359-71.

49. Mandard S, Muller M, Kersten S. Peroxisome proliferator-activated receptor alpha target genes. Cell Mol Life Sci. 2004;61: 393-416.

50. Kliewer SA, Mangelsdorf DJ. Fibroblast growth factor 21: from pharmacology to physiology. Am J Clin Nutr. 2010;91: 254S-7S.

51. Canto C, Auwerx J. Cell biology. FGF21 takes a fat bite. Science. 2012;336:675-6.

52. Houtkooper RH, Pirinen E, Auwerx J. Sirtuins as regulators of metabolism and healthspan. Nat Rev Mol Cell Biol. 2012;13: 225-38.

53. Schmid AI, Szendroedi J, Chmelik M, Krssak M, Moser E, Roden M. Liver ATP synthesis is lower and relates to insulin sensitivity in patients with type 2 diabetes. Diabetes Care. 2011;34:448-53.

54. Cortez-Pinto H, Chatham J, Chacko VP, Arnold C, Rashid A, Diehl AM. Alterations in liver ATP homeostasis in human nonalcoholic steatohepatitis: a pilot study. JAMA. 1999;282:165964.

55. Satapati S, Sunny NE, Kucejova B, Fu X, He TT, Mendez-Lucas A, et al. Elevated TCA cycle function in the pathology of dietinduced hepatic insulin resistance and fatty liver. J Lipid Res. 2012;53:1080-92.

56. Sanyal AJ, Campbell-Sargent C, Mirshahi F, Rizzo WB, Contos MJ, Sterling RK, et al. Nonalcoholic steatohepatitis: association 
of insulin resistance and mitochondrial abnormalities. Gastroenterology. 2001;120:1183-92.

57. Pessayre D, Fromenty B. NASH: a mitochondrial disease. J Hepatol. 2005;42:928-40.

58. Hussain MM, Shi J, Dreizen P. Microsomal triglyceride transfer protein and its role in apoB-lipoprotein assembly. J Lipid Res. 2003;44:22-32.

59. Tiwari S, Siddiqi SA. Intracellular trafficking and secretion of VLDL. Arterioscler Thromb Vasc Biol. 2012;32:1079-86.

60. Gibbons GF. Assembly and secretion of hepatic very-low-density lipoprotein. Biochem J. 1990;268:1-13.

61. Ginsberg HN, Fisher EA. The ever-expanding role of degradation in the regulation of apolipoprotein B metabolism. J Lipid Res. 2009;50(Suppl):S162-6.

62. Kamagate A, Dong HH. FoxO1 integrates insulin signaling to VLDL production. Cell Cycle. 2008;7:3162-70.

63. Tanoli T, Yue P, Yablonskiy D, Schonfeld G. Fatty liver in familial hypobetalipoproteinemia: roles of the APOB defects, intra-abdominal adipose tissue, and insulin sensitivity. J Lipid Res. 2004;45:941-7.

64. Berriot-Varoqueaux N, Aggerbeck LP, Samson-Bouma M, Wetterau JR. The role of the microsomal triglyceride transfer protein in abetalipoproteinemia. Annu Rev Nutr. 2000;20:663-97.
65. Raabe M, Veniant MM, Sullivan MA, Zlot CH, Bjorkegren J, Nielsen LB, et al. Analysis of the role of microsomal triglyceride transfer protein in the liver of tissue-specific knockout mice. J Clin Invest. 1999;103:1287-98.

66. Tietge UJ, Bakillah A, Maugeais C, Tsukamoto K, Hussain M, Rader DJ. Hepatic overexpression of microsomal triglyceride transfer protein (MTP) results in increased in vivo secretion of VLDL triglycerides and apolipoprotein B. J Lipid Res. 1999;40: 2134-9.

67. Cuchel M, Bloedon LT, Szapary PO, Kolansky DM, Wolfe ML, Sarkis A, et al. Inhibition of microsomal triglyceride transfer protein in familial hypercholesterolemia. $\mathrm{N}$ Engl $\mathrm{J}$ Med. 2007;356:148-56.

68. Choi SH, Ginsberg HN. Increased very low density lipoprotein (VLDL) secretion, hepatic steatosis, and insulin resistance. Trends Endocrinol Metab. 2011;22:353-63.

69. Ota T, Gayet C, Ginsberg HN. Inhibition of apolipoprotein B100 secretion by lipid-induced hepatic endoplasmic reticulum stress in rodents. J Clin Invest. 2008;118:316-32.

70. Li JZ, Huang Y, Karaman R, Ivanova PT, Brown HA, Roddy T, et al. Chronic overexpression of PNPLA3I148M in mouse liver causes hepatic steatosis. J Clin Invest. 2012;122:4130-44. 